Meta

Journal des traducteurs

Translators' Journal

\title{
Recueil de connaissances et terminologie pour la modélisation des systèmes de production
}

\section{Daniel Breugnot}

Volume 40, numéro 2, juin 1995

Usages sociaux des termes : théories et terrains

URI : https://id.erudit.org/iderudit/002141ar

DOI : https://doi.org/10.7202/002141ar

Aller au sommaire du numéro

Éditeur(s)

Les Presses de l'Université de Montréal

ISSN

0026-0452 (imprimé)

1492-1421 (numérique)

Découvrir la revue

Citer cet article

Breugnot, D. (1995). Recueil de connaissances et terminologie pour la modélisation des systèmes de production. Meta, 40(2), 284-295.

https://doi.org/10.7202/002141ar d'utilisation que vous pouvez consulter en ligne. 


\title{
RECUEIL DE CONNAISSANCES ET TERMINOLOGIE POUR LA MODÉLISATION DES SYSTEMES DE PRODUCTION
}

\author{
DANIEL BRIEL'Ginot

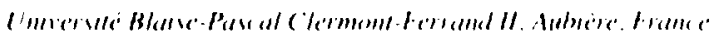

\begin{abstract}
Résumé

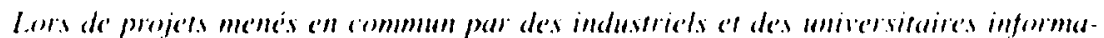

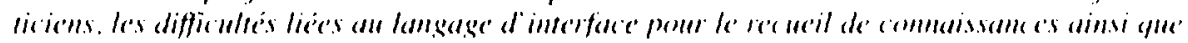

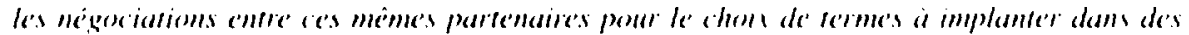

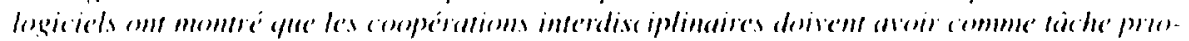

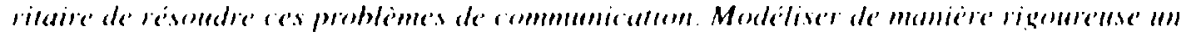

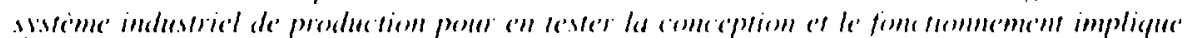

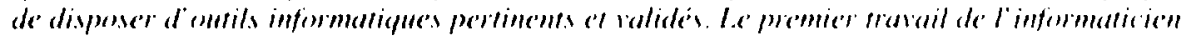

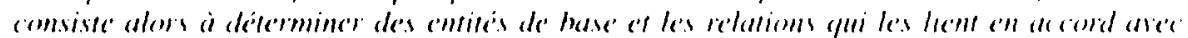

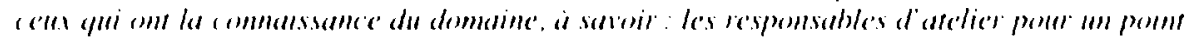

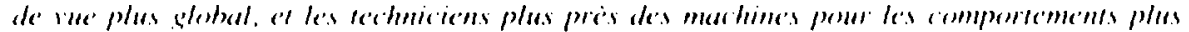

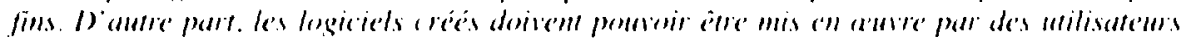

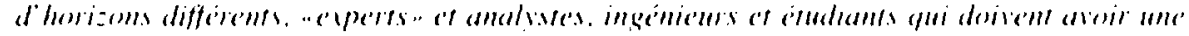

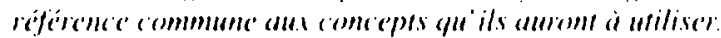

Cel article présente les problèmes rencontrés par une équipe d'informaticiens et l'acquisition de connaissances, dans le domaine de la terminologie des systèmes industriels de production. Les questions posées par l'emploi de langages et de vocabulaires particuliers n'avaient jamais eu pour notre équipe, jusqu ia ce projet, d'importance prépondérante, dans la mesure où les quelques expériences vécues en milieu industriel l'ont toujours été sur des cas très ponctuels, décrits par une seule personne et salns avoir à rechercher des informations sur le terrain auprès d'interlocuteurs divers. II en a été tout autrement pour ce projet proposé par le ministère de la Recherche et de la Technologie. et intitulé "Conception et réalisation d'un environnement de modélisation pour les systèmes d'assemblage" (Gourgand et Kellert $199($ )) qui impliquait comme partenaires des universitaires chercheurs en informatique et des ingénieurs du groupe Valeo, regroupant une vingtaine d'usines fabriquant des équipements d'automobiles. Les systèmes de production modernes sont de plus en plus difficiles à concevoir, à mettre en place el à exploiter. C'est pourquoi, aussi hien durant leur conception yue pour l'amélioration de leurs performances, il devient impératif d'utiliser des techniques de simulation sur calculateur. Le système est donc modélisé et son comportement simulé : il est alors possible de faire varier ses paramètres et de déterminer une configuration satisfaisante, éest-à-dire acceptable à défaut d'être la meilleure. Cette démarche est hien sûr une démarche de spécialiste en modélisation el en informatique. Or, son efficacité à moindre coût n ext possible que si elle est accessible à un responsable d'usine ou d'atelier, appelé expert dans la suite. C"est en effet la personne qui connaît le mieux son système et qui peut déterminer les données les plus pertinentes. Lors d'une première phase de modélisation. l'expert doit spécifier la struclure et le fonctionnement de son système dans un formalisme et avec des entités en accord avec sat vision personnelle. Cette modélisation est 
réalisée avec un environnement informatique qui lui propose des composants de base et des procédures spécifiques. Ces éléments auront été répertoriés, décrits en langage informatique et stockés pour être réutilisés. L'objet de notre projet est précisément la recherche. la spécification et le codage de ces classes d'entités, ainsi que la définition d'une méthode d'analyse et de spécification des systèmes de production avec pour objectifs principaux :

- la réduction du temps d'analyse pour un système donné :

- laugmentation de la fiabilité du modèle:

- la minimisation du nombre des erreurs dues au codage en langage de programmation:

- une meilleure communication entre les personnes impliquées dans un travail de conception-modélisation-reconfiguration.

En résumé. le travail de l'équipe d'informaticiens spécialisés en modélisation est de créer un ensemble d'outils interconnectés (simulateur, système de gestion de bases de données, système d’aide à la décision. interfaces graphiques évoluées...) et une méthodologie d'analyse et de spécification à partir de leur savoir-faire d'une part. et des connaissances recueillies auprès des experts d'autre part. Il faut entendre par expert, une personne qui connaît très bien la structure et le fonctionnement de son système. Il s'agit donc en fait plus de spécialistes. de praticiens (par exemple. un technicien responsable d'une machine) que de personnes faisant autorité dans un domaine donné. Le problème tient à ce que d'une part. les systèmes de production sont complexes (un système de production peut. en effet, être décomposé en un sous-système artificiel et en un soussystème à composants humains), et à ce que d'autre part. il est nécessaire de consulter plusieurs expents appantenant à des sphères d'activité différentes (ingénieurs méthodes, automaticiens, gestionnaires, ingénieurs de production...), avec tous les problèmes de terminologie inhérents à la diversité des compétences. La démarche à suivre consiste donc à rechercher el à structurer toutes les données qui caractérisent une classe de systèmes: c'est la notion de modèle de connaissance décrite par M. Gourgand (1984). Le processus de recherche et de validation de cette connaissance a été globalement le suivant:

- une pré-étude bibliographique des systèmes de production pour en dégager les principales composantes:

- des visites sur sites dont chacun a ses spécificités quant au degré de modernité, aux produits qu'il fabrique, à la complexité :

- des confrontations entre industriels, experts en modélisation, concepteurs d'interfaces homme-ordinateur:

- la mise au point d'un modèle conceptuel des données de type Entité-Association:

- la création et la gestion informatisée d'un glossaire correspondant :

- la diffusion du document dans les différents sites à modéliser, comme élément du guide d'analyse complet, dans un but initial de validation du modèle EntitéAssociation et du glossaire.

\section{ÉTUDE BIBLIOKBRAPHIQUE:}

La façon la plus naturelle d'aborder un nouveau domaine d'étude est de rechercher de la documentation sur tout ce qui se rapporte au sujet. Cette recherche, souvent effectuée par mots clés, ce qui constitue déjà en soi un problème terminologique intéressant. aboutit à sélectionner des publications et des ouvrages de provenances diverses, c'est-à-dire traitant d'un même sujet, mais dans des perspectives différentes. Il va donc sans dire que les termes rencontrés n auront pas les mêmes références, ils peuvent avoir valeur de mots clés dans 
deux domaines différents $\mathrm{n}$ 'ayant d'intersection commune qu'à un niveau d'abstraction très élevé. Même si un cours de philosophie traitant du "concept de modèle» constitue un sujet de réflexion enrichissant. il ne sera pas d'un grand secours, au moins dans un premier temps. pour la résolution d'un problème tel que celui qui nous est posé. Les documents retenus sont donc pratiquement tous issus du seul domaine particulier auquel nous nous intéressons, même si parfois des études réalisées dans d'autrés sphères d'activité pourraient, parr analogié, apporter un éclairage intéressant. Pour un projet ayant pour thème la modélisation des systèmes de production. l'étude bibliographique a évidemment porté d'abord sur les notions de système et de système de production. plutôt que sur celle déjà familière de modélisation.

Lés objectifs de celle pré-étude concernaient trois points principaux, à savoir le recensement de méthodes d analyse de systèmes, la caraclérisation des systèmes à étudier. et la détermination de solutions adaptées aux problèmes à résoudre. Less enseignements apportés par des ouvrages au titre aussi généraliste que "théorie des systèmes et management" ou bien alat gestion par les systèmes" sont certes indispensables pour aborder des systimes complexes comme des ensembles de fabrication automatisés. Cependant la terminologie qu'ils emploient n est pas toujours normalisée, et rarement en accord avec celle employée sur le terrain. l. une des raisons en est peut-être qu il est difficile de spécifier des termes abstraits. Par exemple, le mot stisteme est largement utilisé. et pour le referencer, il est fait appel aussi bien a la cybernétique qu au langage courant. L important pour notre étude est que les définitions proposées ne soient pas en contradiction avec celles concernant une vision plus globale, plus abstraite. Ainsi, le terme systeme d'assemblage' utilise dans le sujet du projet n'est pas en contradiction avec la définition du mot susteme proposée. par exemple, par Bertalanffy $(1968)$, à savoir: "un ensemble d’éléments en interaction orientés vers la réalisation d’un objectif". De même, à ce niveau d'abstraction, la notion très importante de flux est supposée implicite. Ce n'est qu'en descendant au nivealu matéricl d'une usine que l'on sapercevra qu'il existe plusieurs sortes de flux et que leur définition n a rien d"évident. En revanche, les exemples pratiques que l'on peut trouver dans des livres traitant de systèmes de production (Bonetto 1987; Rodde 1989) regorgent de termes techniques n'appartenant qu'au monde des systèmes de fabrication et trop souvent supposés connus du lecteur bien que l'on trouve parfois, en dernière page. un petil glossaire qui prouve que les problèmes de communication dus à la terminologie sont sous-jacents.

La définition rigoureuse de ces termes techniques n'est pas absolument nécessaire à la compréhension des phénomènes exposés. Par exemple. le fait de ne pas savoir exactement ce que significe ke terme palpage dans la phrase "utiliser les correcteurs de la commande numérique après palpage pour tenir comple des défauts de positionnement" $n$ 'est pas un obstacle a la description du fonctionnement global d'un atelier. Tout est affaire de niveau de décomposition: lorsque lon veut comprendre le fonctionnement d'un système. tout ne val pats être modélisé. Suivant le degré de finessie déterminé, les roulements a billes d'un transporteur seront ou ne seront pas pris en compte: de même pour ce qui concerne les opérations effectućes. elles sont affectées d’attributs qui ne les différencient pas forcément aux yeux du concepteur de modèles. Par exemple. des machines effectuant des opérations de cintrage ou de gonflage sur un tube n'ont pas à être détaillées, elles possèdent des caractéristiques semblables, en particulier un temps de traitement. un temps moyen de bon fonctionnement... Cependant. les termes rimtrage et gonflage paraissent connus au lecteur qui se bâtit ainsi inconsciemment une terminologie personnalisée, issue du langage commun. plus ou moins déduite du contexte et approximative: Ie dialogue avec les industriels en apportera la preuve. Dans un premier temps, ces termes servent d'outils de communication et sont mis pour aspération effectuée par la machine en 
question". En revanche. si une analyse ultérieure plus fine nécessite de décomposer cetle machine, les explications d'un expert industriel deviennent nécessaires pour en préciser. sinon en redéfinir, la signification.

Dans cette étape bibliographique, une étude terminologique poussée $n$ 'aurait sans doute que peu apporté dans la mesure où les termes employés sont soit trop généraux, soit dédiés à des cas trop particuliers et donc susceptibles d être remis en cause par les experts du domaine considéré lors des visites sur sites. De plus, la rapidité d'évolution technologique génère une terminologie non normalisée. fluctuante et subjective en rapport avec de nouveaux concepts.

\section{VISITE SUR I.ES SITES INDUSTRIELS}

Les visites sur sites consistent bien sûr à aller chercher l'information sur place. mais aussi à appréhender la réalité du système et à associer aux différentes entités qui le composent les termes des experts et plus particulièrement, les termes proposés par des experts du même système mais de formations et de cultures différentes. On peut noter que dans le cadre de ce travail, la notion d'expert est sensiblement différente de celle courante en terminologie puisqu'il ne s’agit pas là du dépositaire isolé de connaissances de haut niveau. mais plutôt d'un ingénieur ou technicien ayant la responsabilité d'un îlot ou d'un groupe de machines, d'un atelier ou d'une ligne de fabrication. Il a donc une connaissance très fine de l'outil de travail qu'il utilise. Cela justifie que nous nous démarquions de la notion d'expert utilisée en terminologie: la connaissance fournie par le théoricien n'apporte pas l'essentiel pour notre problème, car si sur le papier "lout marche bien", il faut pourtant chercher à comprendre pourquoi sur le terrain "ça marche parfois mal, voire très mal». Donc, une fois dans l'entreprise, au contact de la réalité, qui va-t-on interroger "? Laquelle de ces personnes en costume ou en combinaison de travail apportera les renseignements les plus pertinents ? Posé ainsi. le problème est a priori insoluble.

Le premier interlocuteur est, la plupart du temps, un ingénieur responsable d un atelier ou d'un produit à fabriquer, confronté à des difficultés de rendement ou de reconfiguration. qui est donc plutôt porté à exposer son problème - la plupart du temps en termes de rentabilité - et plus précisément la vision qu'il a du problème qu’à décrire objectivement son système ou à écouter des questions souvent formulées dans un langage qui ne lui est pas habituel. Celui qui possède le mieux la compréhension d'un système est celui qui vit avec. Il est arrivé, par exemple, que deux ingénieurs responsables d'atelier se contredisent au sujet du fonctionnement d'une machine complexe el yue la bonne version soit en fait fournie par le technicien chargé de son fonctionnement. C'est donc au contact des hommes de terrain, chacun ayant une connaissance partielle mais approfondie dans son domaine, qu'on trouvera les informations nécessaires à la compréhension globale du fonctionnement dès lors que le niveau de détail souhaité l'impose. Chacun reconnaît qu il est pratiquement très difficile, pour une seule personne, de bien posséder cette compréhension. Cependant, certains ingénieurs, semblant craindre que la modélisation soit capable de montrer qu ils ne maitrisent pas leur système et de mettre en évidence une certaine incompétence, ont parfois tendance à ne pas comprendre les questions poséces dans un sens objectif et à trier sur des critères subjectifs les informations qu ils fournissent.

Les premiers éléments compréhensibles ne viennent qu'après que l'expert a compris que. pour les besoins de la modélisation. il doit fournir toutes les informations demandées. même si à ses yeux certaines $n$ ont pas d’importance et si elles ne sont pas structurées de la manière dont il les conçoit. Par exemple. pour obtenir des données plus claires et plus exploitables. chaque système est décomposé en trois sous-systèmes interdépendants mais disjoints, à savoir (Breugnot, Gourgand et Kellert 1990a): 
- un «sous-système logique" qui comprend les gammes, la nomenclature, c’esta-dire ce que le système doit fabriquer:

- un asous-système physique» qui est composé par l'ensemble des moyens de fabrication (machines), l'ensemble des moyens de manipulation (transporteurs) ef lensemble des ressources humaines (opérateurs). cest-à-dire avec quoi le système fabrique:

- un «sous-système décisionnel» pour les règles de gestion et de pilotage. c'està-dire comment le système travaille.

Cela oblige lexpert à avoir une vision plus analytique et à ne décrire le fonctionnement de son système qu avec des notions clairement reconnues. Il ne s"exprimera donc plus tout à fait dans son langage habituel el même si ce n est avec une terminologie bien fixéce. il le fait au moins avec des concepts aux contours bien définis. En fait. la première étape consiste à définir des classes a partir des objets du monde réel. et en fonction de notions générales comme phase de lranspont ou valew ajoutét'. On regroupera par exemple tous les robots, bras manipulateurs, stations élévatrices.... dans le même ensemble. Même si un tel classement n'est pas très rigoureux êt est appelé à être modilié, il pernnet néanmoins de decrire le système at son fonctionnement. les entités détinies par cette aterminologie intermédiaire" représentent la partie statique de la description. La partie dynamique. le fonctionnement, est plus lice au langage, à la laçon qu a l'expert de s'exprimer. Pour clarifier et fixer la description du fonctionnement, des outils de spécification spécialisés (souvent issus du monde de l'automatique et de l'informatique, tels que graphes, tables de décision, organigrammes, réseaux de Petri...) seront utilisés par la suite pour éviter les imprécisions et les déviations dues au langage naturel el pour structurer la complexité des règles. Cela permet d'éviter par exemple l'un des dialogues restés célébres de cette étude :

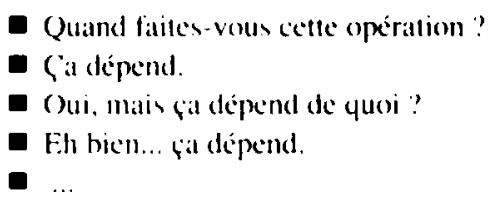

Ce genre de réponse est typique de l'aspect diffus de la connaissance. Quand les règles sont simples, du type Si condition Alors action. leur expression est claire: en revanche. lorsqu elles sont très complexess, comme par exemple dans les systèmes très automatisés où la réalisation d'unce action dépend des valeurs de nombreux paramètres, il est très difficile de posséder la compréhension globale du fonctionnement du système. De plus, personne ne sexprime jamais en disant: "Si la condition 1 est remplie, si la condition 2 est remplie et si le parametre $n$ est plus petit que 5 et plus grand que l. alors les actionneurs cl el c2 sont activén’. mais plutôt adans certains cas, on fait fä et dans d'autres, on active ces deux actionneurs". Donc. même lorsque le fonctionnement du système est délerministe. et a priori descriptible de façon claire. l'expert $n$ 'arrive pas à exprimer simplement le modèle qu’il sen fait. la façon dont il le perçoit.

Le but principal de ces entretiens n était cependant pas l'analyse tine de chaque site, mais le recueil des éléments de base nécessaires a la construction de l'environnement de modélisation pour lequel divers groupes de travail ont à collaborer.

\section{CONFRONTATION}

l.etape precedente. a savoir les visites sur sites, a permis la constitution d'un recueil 
d'informations jugées pertinentes, qu'il s'agiı non pas de normaliser, ce qui serait trop ambitieux, tout au moins à ce stade. mais plutôt d'harmoniser, de fixer et de rendre commun à tous les participants du projet. Sachant qu'il est toujours difficile d'organiser une réunion de travail avec beaucoup de participants. la première difficulté a consisté à trouver une perspective commune aux trois groupes présents que sont:

- les experts industriels ayant une connaissance fine de leurs systèmes et des nombreux problèmes qu'ils leur posent :

- les analystes qui cherchent à extraire et à structurer cette connaissance pour pouvoir appliquer des méthodes de résolution:

- les concepteurs de logiciels, dont le but est d'écrire rapidement des programmes exploitables en éludiant le moins possible les systèmes étudiés, mais en répondant aux besoins spécifiés.

Chaque intervenant a tendance à considérer son domaine d'activité et a fortiori sa terminologie usuelle comme étant unique et connu des autres. De plus, la vision que chacun a d'un même objet est différente. Si l'on prend pour exemple un comroyerur à accumulation, il sera caractérisé par des attributs particuliers à chaque domaine, ou d'importance différente pour chacun d'eux : l'expert industriel pensera d'abord au coût. à l'encombrement et à la vitesse. le concepteur de modèle prendra d'abord en compte la vitesse, la capacité maximale (le nombre de pieces qu'il peut supporter suivant les types). les lemps de panne et de reconfiguration qui sont des paramètres habituellement retenus pour ces études bien que la pertinence de ces paramètres en fonction des objectifs de la mockélisation ne soit pas a priori évidente. En ce qui concerne l'interface utilisateur, ce convoyeur sera défini d abord par des données graphiques de représentation à l'écran. qu élles soient statiquess ou dynamiques, et par des grilles de saisie associées.

La principale conclusion de la première réunion fut donc qu'il était nécessaire de définir un lexique de références comme moyen d'échange possible, surtout si l'on considère que le langage dit "naturel" est lui aussi fortement typé et que, par exemple. la description du fonctionnement dynamique d'un système manque parfois de rigueur et qu'elle est souvent plus sensitive qu'analytique.

De la même manière. un spécialiste en modélisation a tendance à voir les choses sous un angle qui facilitera la spécification d'un modèle: là où un industriel voit un convoyeur à rouleaux où sont installés des capteurs, le concepteur de modèles considère des tronsons de comroverw, notion qui n'a aucun sens pour un ingénieur. En revanche, la notion de troncoon de chemin qui est une entité paraissant plus naturelle a été admise par tous les partenaires. Le but de cette étude étant de mettre des techniques particulières à la disposition d'un nombre important d'individus, il est impératif que les premiers contacts entre le produit offert et l'utilisateur moyen se réalisent le plus naturellement possible. et suscitent un intérêt pour la suite. C'est la raison pour laquelle les solutions purement néologiques ont été éliminées. Dans notre contexte, "purement néologique» signifie qu il n’était pas question de créer un nouveau mot, en revanche, si un désignant composé tel que tromson de chemin cité précédemment peut être qualifié de néologisme. il est pourtant perçu plus de façon linguistique que terminologique par les experts. dans la mesure où la notion désignée est reconstituée sans ambiguïté à partir des deux composants. C"està-dire que non seulement la signification évaluée est celle qu'a voulue le concepteur, mais la relation de composition entre chemin et tronson de chemin laisse sous-entendre qu'il existe aussi une relation de composition pour la modélisation.

Chacun des termes propres au nouveau domaine a été choisi en quelque sorte statistiquement : ou bien la notion en question ne prête à aucune discussion et le terme habituel 
correspondant convient, ou bien cette notion est peu précise parce qu'elle n'a besoin de l'être que pour la modélisation et moins en milieu industriel. II est à noter que la description de la partie physique des systèmes de production ne pose pas de problèmes particuliers, si ce n'est une classification; en revanche, tout ce qui concerne la partie logique. le fonctionnement. est beaucoup plus délicat à spécifier. Mais dans ce cas, et en accord avec les experts, le terme choisi, même s'il n'est qu'approximatif' doit évoquer le concept désigné et sa définition sera précisée dans le glossaire du guide d'analyse. La première dénomination de tronson de chemin était sous-chemin, mais la relation de chemin à sous-chemin était trop mal perçue, un chemin étant défini comme la matérialisation d'une transition entre deux entités, et la notion à définir comme une partie de chemin indécomposable, caractérisée par le type de transport qui y est utilisé. Une autre suggestion. à savoir chemin affecté pour indiquer qu'un moyen de transport particulier était affecté à cette portion de chemin $n$ 'a jamais trouvé d'écho favorable che\% les industriels.

À ce stade. l'ensemble des termes répertoriés. c'est-à-dire recensant toutes les classes d'entités existantes dans un système de production pour une vision de modélisation. a toule la cohérence souhaitable puisque. pour chacun deux. les personnes concernées conçoivent soit la même chose, soit des entités reliées de manière unique. Il est à noter que le premier glossaire comprenant 1.34 détinitions n a élé admis comme document de travail qu'au bout de huit mois de discussions. Les termes de ce glossaire sont définis d'une façon qui ne satisferait sans doute pas complètement un terminologue : il manque des références. les définitions ne sont peut-être pas rédigées de manière assez précise. mais l'important ici est moins d'établir une fiche terminologique que de mettre au point un ensemble cohérent d'entités qui serviront à décrire un système industriel de production.

\section{STRIC"TURATION DE LA CONNAISSANCE}

Pour que chacune de ces entités puisse être considérée comme pertinente pour la construction d'un modèle, il ne suffit pas qu'elles soient rangées dans un dictionnaire par ordre alphabétique, mais il faut mettre en évidence les relations qui les lient les unes aux autres. Au fur et à mesure que de nouveaux systèmes seront analysés, la structure de l'ensemble des concepts détinis sera testée, éventuellement modifiée, et petit à petit stabilisée el validée. Parmi les nombreux modèles proposés, le modèle Entité-Association de Chen (1976) a été choisi car il offre le meilleur rapport de la puissance de description sur la simplicité de la représentation. Cet outil. conçu initialement pour modéliser des bases de données. s'est avéré très bien adapté à la représentation et à la structuration des connaissances pour les systèmes de production. Il s'inspire de concepts proposés par la théorie des systèmes. La définition de concept utilisée ici est celle du dictionnaire. c'està-dire une représentation intellectuelle d'un objet conçu par l'esprit et ne se différencie pas, pour notre propos de celle de notion. Ces objets sont définis pour permettre une représentation d'un système. Ce sont les concepts d'entité, d'association entre deux entités ou plus et d'attributs qui sont la propriété d'une entité ou d'une association. Un typé d'association est caractérisé par les cardinalités des typess d'entité. Cardinalité a ici la même définition qu'en mathématiques, à savoir le nombre d'éléments d'un ensemble - on parlera aussi d'occurrences: il s'agit des valeurs possibles du nombre d'entités prises en compte dans lat relation. Ces cardinalités ajoutent des contraintes et complètent la définition des entités qui figurent dans le glossaire.

Le formalisme graphique hahituel est celui de la figure 1 (pour des questions de lisibilité, les attributs $n$ apparaissent pas toujours dans ces schémas). Cet exemple indique que lentité unité de production ou de storkage' qui est l'entité englobante pour cette classe de systèmes peut, par exemple, comprendre zéro machine (c'est le cas d'une unité 
de stockage) ou plusieurs machines: à l'inverse, une machine appartient à une et une seule unité de production ét de sfockage. Dans les cas pratiques, toutes les entités du schéma n'ont pas le même poids ou le même degré d’abstraction ou de généricité. Par convention, les entités de la partic supérieure sont les plus génériques comme dans l'entité unite' de producfion e' de stockage de la figure 1, de manière à représenter des relations hiérarchiques que le schéma Entité-Association ne prend normalement pas en compte mais qui sont nécessaires pour l'analyse d'un système, qu'elle soit montante con part des éléments particuliers de base pour créer des ensembles ou classes intermédiaires et arriver à une entité qui ènglobe le tout) ou descendante. Les problèmes de terminologic se posent aussi. évidemment. dans ces niveaux intermédiaires où il a fallu créer de nouveaux ohjets en relation avec la méthodologie de modélisation.

Un exemple de l’intérêt des cardinalités associées à une relation est, sur la figure 1. de différencier les notions de poste et de sfarion. Ces deux éléments sont similaires, à ceci près qu'un posté. à l'inverse d'une station, requiert la présence d'au moins un opérateur. ce qui peut se lire sur le schéma par: un élément du groupe station nécessite zéro ou n homme et un élément du groupe poste nécessite un ou $\mathbf{n}$ homme. Un autre exemple est celui de la différenciation des notions de tramsporfeur sfatique et ramsporteru dimamique qui sont nécessaires pour la modélisation. mais pas forcément très claires pour un expert industriel qui a l'habitude de désigner plutôt des occurrences de ces types d'entité, par

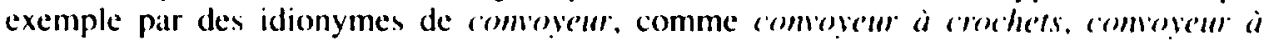
hande. commoyeur à chaine'.. ou bien par des noms de marques comme Clark. Fonwick... ou bien encore par des termes dérivés de chariol tels que chariot cilélateur, chariof filoguidé. etc. (e sont les propriétés de ces objets. à savoir leurs attributs et la manière dont ils fonctionnent. mais aussi la façon dont ils sont reliés à d'autres par le lype d’association et les cardinalités, qui vont définir s’ils appartiennent à la classe des rarsmsporterirs statiques, ou à la classe des ranspontews dymaniques. Dans le glossaire. le terme a miori surprenant de tramsporterw statique est défini comme «un moyen de transport immobile (par rapport au sol sur lequel il repose) auquel est dédié un ensemble de tronçons disjoints»» et un transportear dymamiche comme "un moyen de transpont qui emprunte un ou plusicurs tronçons définissant le routage" (le terme "moyen de transport" a été précisé par ailleurs et les définitions données ici pourraient être plus détaillées à condition d'être healucoup plus longues). Les deux transporteurs ont les mêmes attributs, mais la différence de cardinalité dans le schéma Entité-Association de la figure 1. à savoir qu un tronçon ne peut supporter qu un seul tramsporle'tr statique mais plusieurs transporlews dinamiques, indique que le premier occupe en permanence un emplacement physique, alors que le second ne l'occupe que le temps de son passage. On indique ainsi de manière très concise une différence essentielle atu moins dans le domaine de la modélisation. Bien sûr. l'ensemble des entités. les relations et les cardinalités sont définies et reliées en accord avec les experts industriels, seul le formalisme est propre aux informaticiens.

On constate qu'avec cette façon de contraindre les définitions proposées, le concepteur de modèles est assuré, par les termes qüil emploie. de ne manipuler que des ohjets validés (Breugnot, (iourgand et Kellen 199()h). Il est à noter qu'il s'agit de la définition de classes d'ohjets, et que la décision d inclure un élément particulier à telle ou telle classe est à prendre par l'expert, lors de la modélisation. La plupart des cas sont résolus de manière quasi implicite, mais certaines entités ont des caractéristiques qui permettent de les rattacher à un ensemble ou à un autre. 


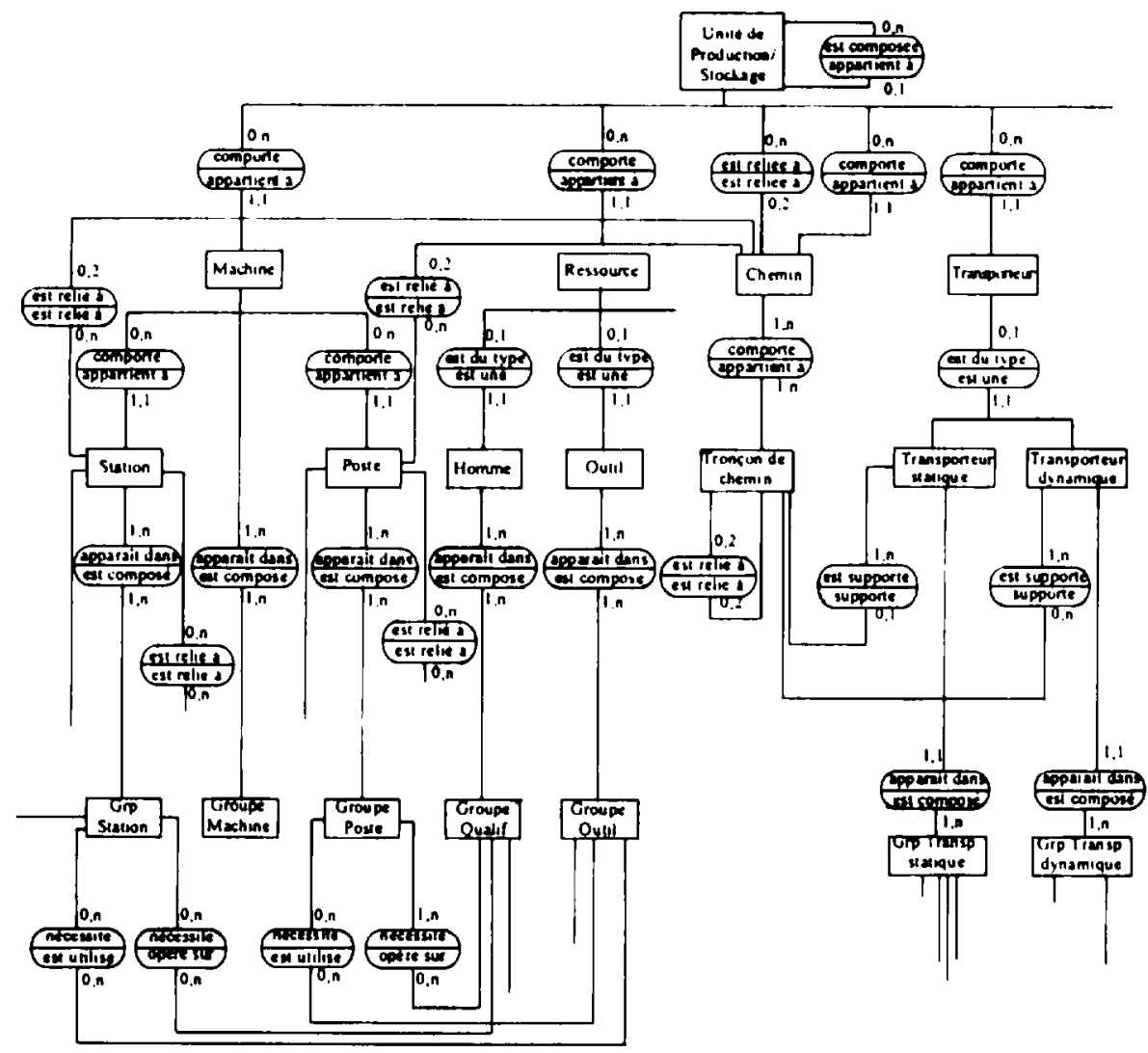

Figure 1:

Vue partielle du modèle Fintité-Association pour les systèmes de production (Breugnot 1990b)

Le modèle Entité-Association qui a été construit peut être complété pour un point particulier, lors de la modélisation deun système possédant des caractéristiquess non encore répertoriées. Cependant, il n'a pas été mis en défaut par la quinzaine de systèmes modélisés depuis sa mise au point. Bien que la stabilité de l'ensemble soit assuréce en particulier par le nombre important de cas réels étudiés. il a semblé intéressant de pouvoir disposer d'un outil informatique qui puisse faire la mise à jour du schéma et la modification automatique de la partic correspondante du glossaire. De toutes façons, ce schéma est appelé à Evoluer, ne serait-ce que par l'apparition de nouvelles entités lechnologiques qu il faudra intégrer dans les systèmes existants.

\section{DIFFUSION F.T BAN('S I)ESSAIS}

Les différentes phases d'acquisition, de mise en fonne et de validation de la connaissance nécessaire à la modélisation des systèmes de production sont schématisées dans la fïgure 2. La première ćtape repose essentiellement sur le dialogue avec les experts et propose une méthode et des outils pour un domaine en fonction des objectifis de 
modélisation définis en commun. Les étapes de vérification et de validation consistent. en étudiant des cas réels, à vérifier que tous les éléments du domaine ont été catalogués et que la façon dont ils sont pris en compte pour la modélisation est correcte.

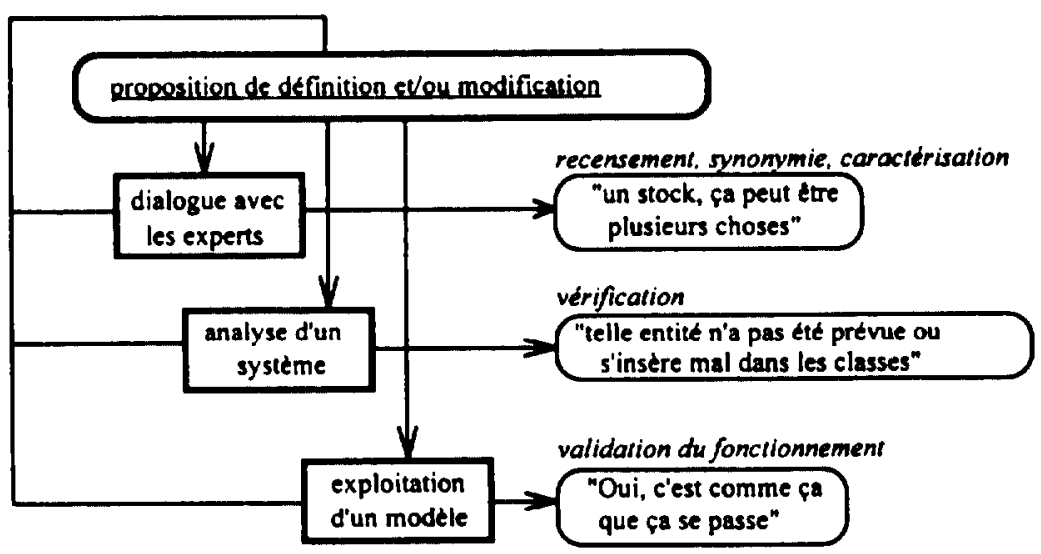

Figure 2:

Les difrérentes phases d'acquisition et de validation

Donc, pour s'assurer que les résultats du travail effectué peuvent être utilisés efficacement dans la conception de l'environnement logiciel, et rentabilisés par les utilisateurs potentiels, un guide d'analyse accompagné du glossaire a été diffusé dans les sites industriels dès sa première version stabilisée, avant même qu un prototype du logiciel soit terminé. Le but de ces bancs d'essais est de tester la robustesse des définitions des entités du schéma de données. à le compléter. et à vérifier l'adéquation de la méthodologie d'analyse proposée à la résolution d'un problème de modélisation pour un exemple appartenant a la classe des systèmes d’assemblage. À ce stade, les populations concernées élaient de deux types: des ingénieurs des entreprises industrielles, et des étudiants en informatique pendant leur stage en entreprise. Pour les premiers, familiers des systèmes de production. il s'agit de filtrer et de mettre en forme leurs connaissances en accord avec les notions qui leur sont fournies. Pour les seconds, ayant déjà suivi des cours de modélisation. la difficulté consiste à rechercher les informations pertinentes à partir des entités du schéma de données. Le guide d'analyse et le glossaire définissant les entités de l'environnement constituent un cadre rigide qui, en caricaturant, évite à l'expert de modéliser n'importe comment et à l'informaticien de modéliser $n$ 'importe quoi. Dans tous les cas, le temps nécessaire à la modélisation d'un cas particulier a été pratiquement diminué d'un facteur dix.

Toutes les personnes impliquées dans ce projet, de la pré-étude à la validation. constituent une population relativement importante et disparate, à savoir six chercheurs universitaires, douze industriels, huit ingénieurs de sociétés de service en informatique et une douzaine d'élèves ingénieurs. Tous n'étaient pas concernés par les mêmes problèmes et. en raison de leur nombre, on peut donc estimer que les termes liés aux concepts et définis pour analyser un système en vue de sa modélisation sont maintenant bien stabilisés. 


\section{CONCLUSION}

Tout ce qui vient d'être écrit expose, non pas la façon d’aborder et de résoudre des problemes de terminologie en général. mais la prise de conscience de tels problèmes au travers d'une expérience de travail en commun sur un objectif particulier par des spécialistes de domaines différents. Le but principal est d'abord le recueil de la connaissance spécifique à un domaine, celui des systèmes de production, et sa mise en forme en fonction de critères d'un autre domaine : celui de la modélisation informatique. C"est un problème à caractère général sur lequel réfléchissent de nombreux chercheurs. La façon dont il a été mené apporte des résultats satisfaisants. comme le prouvent les nombreux bancs d'essais effectués en entreprise. Flle est actuellement reconduite pour l'étude de systèmes autres qu“industricls.

Lil structurattion des connaissances s'appuie sur des outils tels que le modèle EntitéAssociation, developpés pour et par le secteur informatique des systèmes d'information mais. comme l'indique $\mathrm{F}$ : Gaudin (1990), la convergence existant entre les problèmes de la modélisation de la connaissance et ceux de la terminologie implique le développement de coopérations interdisciplinaires.

Lors de ce projet. la plus grande part de temps a été consacrée aux entretiens. pour le recueil d informations proprement dit, el pour leur harmonisation. Il est clair qu un tel travail hasé uniquement sur des documents sur papier n'aurait pàs pu aboutir: c'est grâce a la diversité des points de vue que le recensement effectué a été très complet, même au prix des difficultés liées aux problèmes de langages. Ces problèmes sont inévitables dans la mesure où un expert ne peut décrire en particulier l'aspect dynamique, vivant, de son système qu avec le langage qui lui est habituel. I.ui en imposer un autre conduirait à séloigner de la réalité telle qu il la perçoit.

La complexite de cet aspect dynamique est due aux fortes comélations existant entre les entités de cette classe de systèmes. et pousse à insister sur la nature de ces relations. Quel rapport existe-t-il entre celte conception, essentielle pour l’informaticien. et celle de système notionnel utilisée en terminologiè ?

Comme nous lavons vu plus haut, les discussions concernant la détermination de dénominations jugées pertinentes ont été nombreuses et longues, chaque intervenant faisant des propositions en fonction de son acquis culturel et de son environnement de travail. Cependant, le fait que certaines de ces dénominations aient dû être modifiées au cours du temps montre que, dans l'optique qui était la nôtre, une volonté normalisatrice n'aurait pas été appropriéc. De plus, dans des domaines de spécialité à l'évolution rapide. les choses naissent. se développent et meurent souvent trop vite pour qu une norme puisse entrer dans les faits.

Les intervenants de ce projet. concepteurs et utilisateurs sont tous de langue française. Pour réaliser des versions dans d'autres langues du guide d'analyse et de l'interface utilisaleur du logiciel se pose le problème de savoir si une traduction de type classique suffira, ou si des entretiens similaires à ceux qui ont été exposés ici devront être conduits avec des experts dans les pays concernés. Il s'agit là non pas de mettre au point des notions elles sont déjà détinies et stockées dans une base de données. en l occurrence MC4 de J. Henning (1989) —. mais de trouver des dénominations en accord avec lés caractéristiques sociales et linguistiques locales.

le probleme essentiel reste celui d'une modélisation du monde qui nous entoure, suivant des criteres particuliers et en fonction du savoir d'individus de cultures et de langages différents et de leurs besoins. 


\section{RÉFERENCES}

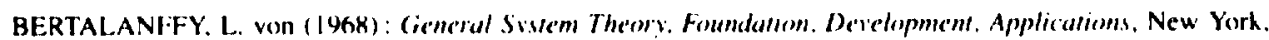
(i. Braciller.

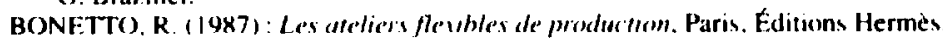

BRE:IGINOT: D., GOURGAND. M. et P. KELLERT (IYq)a): “SIGMA: An Intelligent and Graphical Environnent for the Modelling of Awembly-Systems", International Conference on Operation Research. Viennt. Autriche. 28-31 atoût 1990).

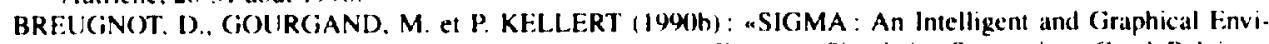
ronment for the Moxkelling of Flexible Asxembly-Systemsm, European Simulation Symposium, Gand. Belgiyue, $x-10$ novenibre $19 \%(0$.

CHEN, P. P. (1976): : The Entity-Relationship Model. Towards a Unified View of Data", ACM Tramsoctions $a m$ Datathase Siskems, vol. I. nก I. pp. 9-36.

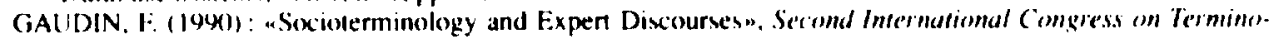
legy and Kinutedge Engune'ring. Trier. Allemagne, 2-4 (xcobre 1490.

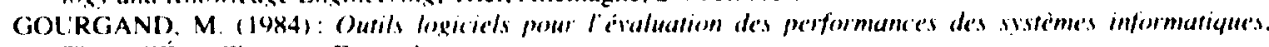
These d'Ftat, Clermonl-Ferrand.

GOURGANI), M. et P. KELLERT ( 199() ) :Conception et réalisation d'un outil de modélisatton de l'assemhlage". Rapport de fin de contral MRT

HENNIN(;, J. (1989) : "MC4 : A Package for the Management of Terminological Data". Conference on Terminology in Advanced Microcomputer Applications, organisée par International Network for Terminology. Vienne. Autriche, 22-23 nov. 1489, IVersion trangaise "Exemples de continuite dans la gestion atutomatique de données terminologiques". Mi'a, vol. 34, n"3. pp. 493-445/.

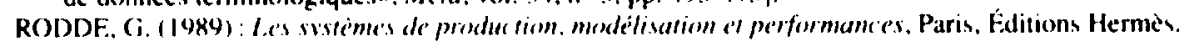

